\title{
Mechanical triggering of Wnt3a and beta-catenin in 3D bioreactors: The role of frequency and rhythm
}

\author{
Máximo-Alberto Díez-Ulloa ${ }^{\text {* }}$ and Ramiro Couceiro-Otero ${ }^{2 *}$ \\ ${ }^{1}$ Orthopaedics Service, Universitary Hospitalary Complex of Santiago de Compostela, Spain \\ ${ }^{2}$ Medical Translational Oncology Laboratory, IDIS, Santiago de Compostela, Spain
}

\begin{abstract}
WNT/Beta-catenin pathway plays a key role in stem cell differentiation as well as other biological processes such as cell adhesion, proliferation or even invasion in tumor microenvironments. Cells receive different inputs from the surrounding conditions, either biochemical or physical cue, such mechanical stimuli represents an interesting regulatory mechanism due to its correlation with physiological conditions. In this work we describe how stem cells cultured on a scaffold in a bioreactor are able to modulate their response to a cyclic flow translating it into a differential expression of WNT3a and beta-catenin. During this study we found that frequency of stimulation influence the expression of Wnt-3a and Beta-catenin expression in a timely fashion while also being affected by the duration of the stimuli, together, it was also found that there is a threshold for both mechanical inputs which clearly mark a higher gene expression of WNT-3a and beta-catenin.
\end{abstract}

\section{Introduction}

Mesenchymal stem cells (MSCs) are defined by a clonogenic capability as well as the ability to differentiate into several cell types. MSCs are able to remain long quiescent, activated and return to a quiescent state without phenotypic changes which might lead into a malignant transformation.

Cellular homeostasis is regulated by tow mechanisms, the first one being an autocrine, paracrine and endocrine based one where all biochemical molecules interact with activate receptors (usually in the membrane) triggering the activation of different pathways. On the other hand, intercellular communication is essential for maintaining tissue homeostasis and, therefore, the organic and systemic.

The second mechanism relies on pure mechanical stimuli to which cells are sensitive; these stimuli regulate cell adhesion (of great interest in the tumoral pathology, described for the first time by Curtis [1], cell proliferation and differentiation. In English the word "cue", which are words or phrases said to an actor during a theatre play to remind him about his role, i.e. the indication to trigger an action; this concept seems especially interesting.

These metabolic pathways triggered by mechanical stimuli could be translated through a tensegrity model (Ingber), which gives a new dimension to the role of the interrelation of cells and extracellular matrix. These mechanical signal transduction will translate physical signals into biochemical activators (ion channels, the FA kinase, protein G, via MAPK (2 BMP-related), RhoA) and will exert a cytoskeleton regulation which will ultimately change shape and cell volume leading to a differential gene expression in response to an external mechanical stimuli. Several pathways have been correlated with the tensegrity model [2] of special interest in the musculoskeletal is the the Wnt pathway, described by Nüsse et al. in 1984 [3].

This mechanobiological models have yielded lots of information related to Wnt pathway stimulation, not easy to synthesize as a body of knowledge. By way of summary, it is known that: a) if there is o repeated stimulation in time, it is more effective than isolated spot; b) if there is some rest as recovery time from hour and up to three, there is a bigger effect afterwards; c) forces can vary from tension to compression, but, above all, it has been studied uni or biaxial deformation by stretching through tensional forces (cells cultured on membranes which stretch periodically in one or two axes), these tensions generated a cell deformation, although the effect of shearing has also been studied by fluids (role of the Cilia of membrane) and vibration (loudspeakers) that generates energy without acceleration or deformation of the cells; d) the response may be enhanced or diminished by specific molecules present in the environment (eg. TGF - beta and enhancers of chondrogenesis).

However, at the present time some questions still remain unknown regarding this initiation of Wnt /beta-catenin pathway, and thus, the switching to MSC towards osteogenesis instead of adipogenesis: a; amount of load (force that produces a deformation) necessary to produce any effect, $b$; the role of: b1; the frequency, b2, or rhythm (variation of frequency) or b.3) the duration of this mechanical stimulus, or even if these are variables with any significance at all. It is not known whether there is a minimum threshold required to trigger response or if any mechanical stimulation, whatever its magnitude produces such

Correspondence to: Máximo-Alberto Díez-Ulloa, Orthopaedics Service, UHC Santiago de Compostela, Trav. da Choupana s/n 15706 Santiago de Compostela, Spain, E-mail: maximoalberto.diez@usc.es

Ramiro Couceiro-Otero, Translational Medical Oncology Laboratory, IDIS. Santiago de Compostela, Trav.da Choupana s/n 15706 Santiago de Compostela, Spain, E-mail: ramiro.couceiro.otero@sergas.es

Key words: stem cells, WNT, beta-catenin, scaffold, bioreactor, tensegrity

Received: December 09, 2015; Accepted: January 02, 2016; Published: January 07,2016 
an effect. With this state of knowledge, we set the following hypothesis.

Pure mechanical stimuli may initiate the Wnt/Beta-catenin pathway and, besides, this pathway is sensitive to variations in rhythm and duration of such a stimuli, probably with a threshold effect below which no response will be triggered

In this present experimental work we try to correlate physical stimuli with changes in WNT3a and beta- catenin levels of expression and its connection with mechanosensing.

\section{Material and methods}

\section{Cell culture}

Mesenchymal stem cells were obtained from patients undergoing a regular surgical procedure at the department of Orthopaedic Surgery. The procedure fulfilled the protocols approved by the local Ethics Committee. MSCs were isolated by means of a Histopaque gradient as described before by Gnecchi and Melo [4]. Briefly, aspirated bone marrow is slowly pipetted on top of a histopaque volume (50:50) and immediately centrifuged at $2000 \mathrm{rpm}$ for 30 minutes. The central layer containing MSCs is separated and centrifuged for another 15 minutes at $700 \mathrm{rpm}$ in order to discard any remaining ficoll. The remaining pellet is suspended in DMEM supplemented with 20\% FBS and Pen/ Strep. Cells are left to grow until confluence is reached.

\section{Scaffold fabrication}

Hydroxiapatite (HA) scaffolds were fabricated using a robocasting technique at the University of Santiago de Compostela in a wood pile pattern with a pore size of 180 microns. HA was obtained from Keramat (Ames, Spain). Robocasted pieces were later on processed at $900^{\circ} \mathrm{C}$. All the pieces were autoclaved before proceeding with cell culture (Figures 1 and 2).

\section{Bioreactor culture}

Bioreactors were acquired from EBERS. Cells were grown on the HA scaffolds until confluence was reached (Figures 3 and 4 ) and placed in a central chamber where different rates of flow were exerted. A peristaltic pump (Ismatec) was used to flow cell culture media through the chamber leading to a maximum pressure of $0.68 \mathrm{~Pa}$. A cell density of $7 \times 10^{4}$ cells $/ \mathrm{cm}^{2}$ was used throughout the experiments.

In order to trigger a dynamic response from the cultured cells, cyclic flow pulses were applied to the collagen surfaces. In brief, the HA scaffold was compressed for a period of 5 minutes with 1, 5 and 15
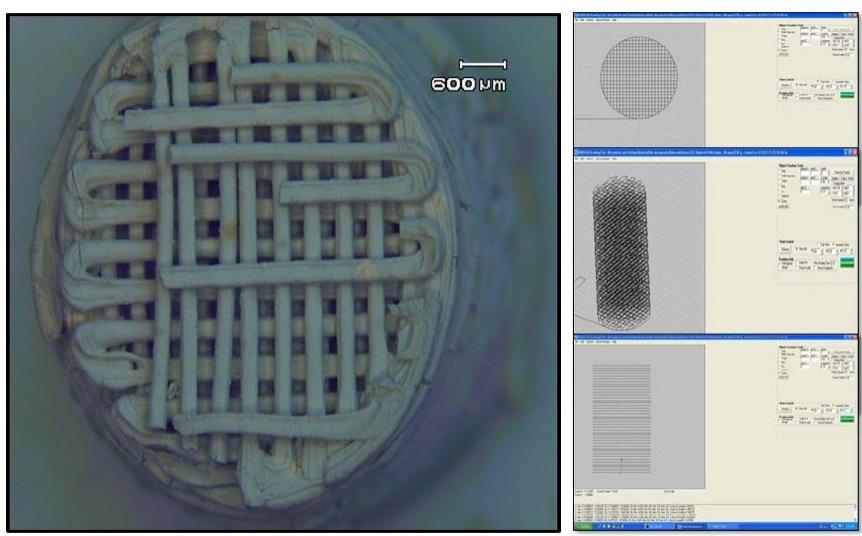

Figure 1. Scaffold design and manufacture before thermal treatment.

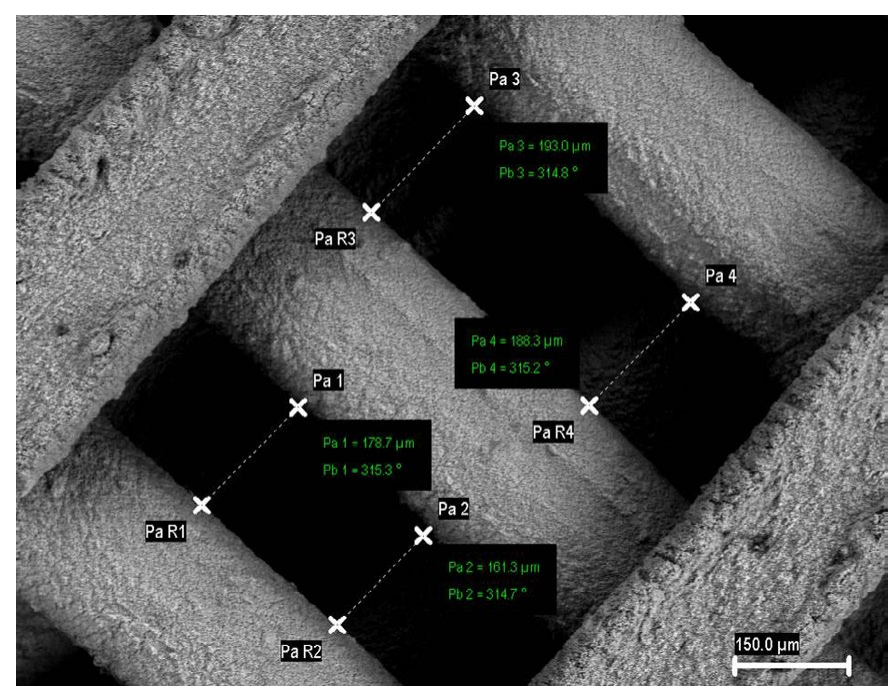

Figure 2. Hydroxyapatite scaffold manufactured by robocasting. Pore sizes were measured by SEM.

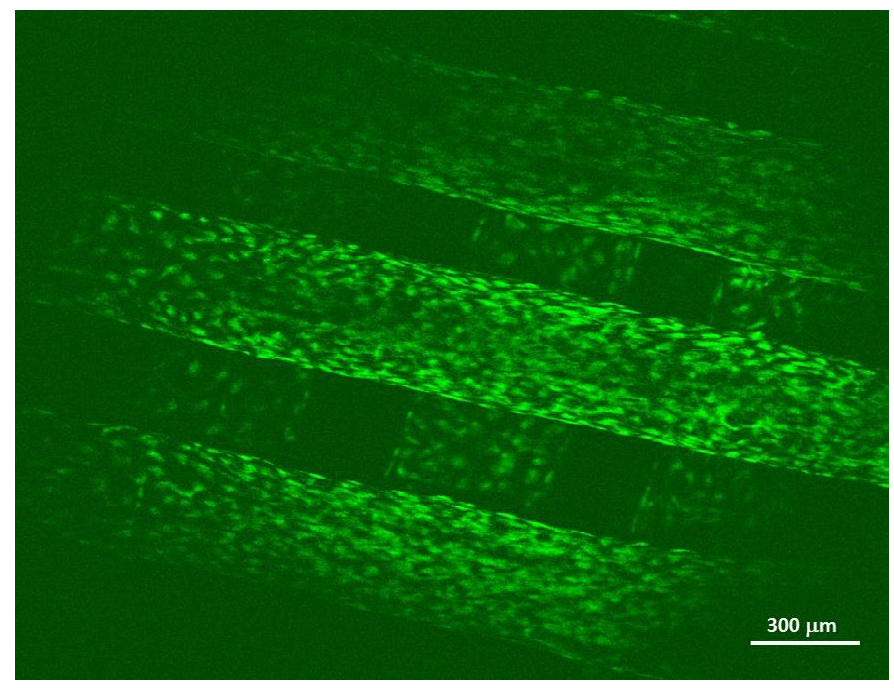

Figure 3. Mesenchymal stem cells cultured on a HA scaffold. Cells were stained with Calcein-AM and Propidium Iodide. Green signal shows a good survival rate for this culture system.

cycles per minute, another group used 15 cycles per minute during a timespan of 5, 40 and 90 minutes.

\section{Gene analysis}

In order to obtain an idea of how WNT/Beta-Catenin was being mechanoregulated, the following WNT3 and beta Catenin primers were purchased from a local distributor (Costoya SL): Wnt3a F 5' CACCACCGTCAGCAACAGCC 3';

Wnt3a R $5^{\prime}$ AgGAGCGTGTCACTGCGAAAG 3'. Betacatenin, forward primer $5^{\prime}$-gtgctatctgtctgctctagta- $3^{\prime}$, reverse primer $5^{\prime}$-cttcctgtttagttgcagcatc- $3^{\prime}$ and tagged with SYBER Green.

\section{Confocal microscopy}

Cultured scaffolds were taken to a Leica SP-2 confocal microscope and cells stained with calcein-AM/ Propidium Iodide to get and insight of cell distribution in static conditions. 


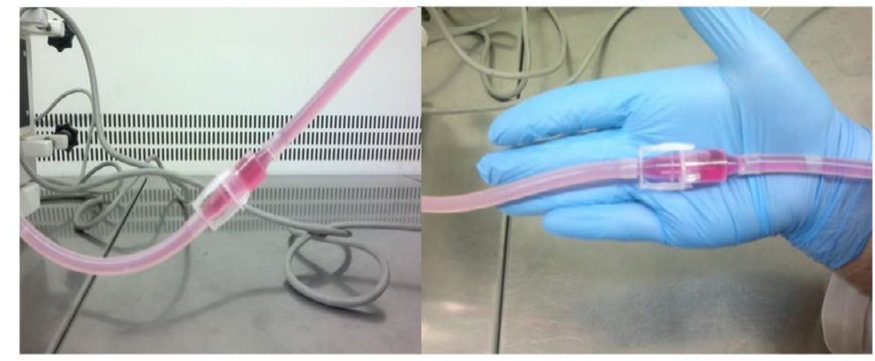

Figure 4. Bioreactor set-up. The flow chamber contains the scaffold cultured with mesenchymal stem cells and D-MEM is flown through it.

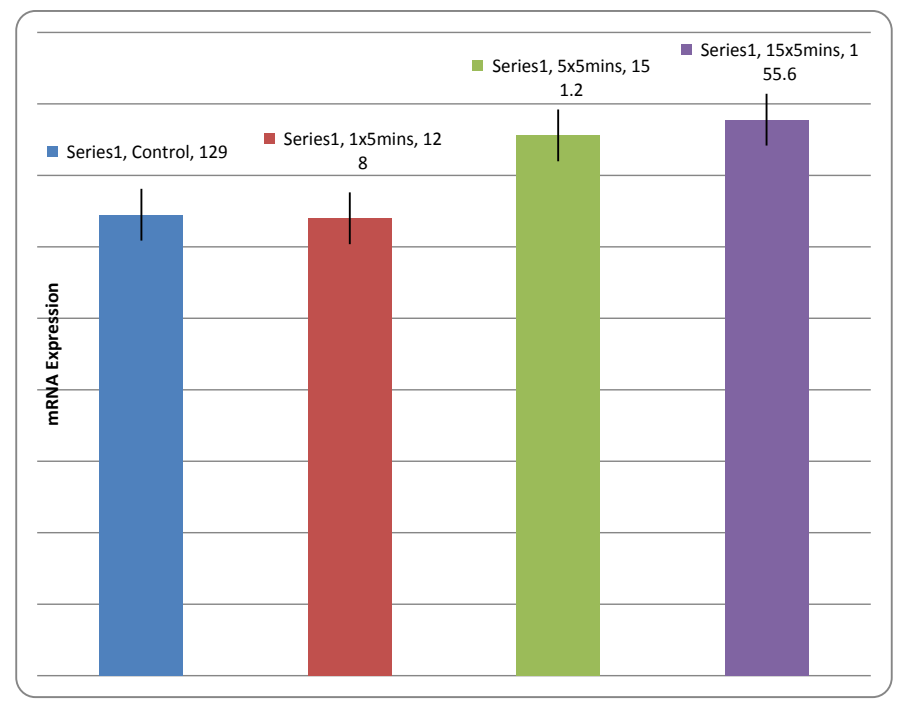

Figure 5. WNT3a Expression. A higher mRNA is found at the $15 \times 5$ minutes group. T-Student test $\mathrm{n}=8$ and $\alpha=0.05$, H0: $\mu 1=\mu 2 ; \mathrm{H} 1: \mu 1>\mu 2$. P-values for the $5 \times 5$ and $15 \times 5$ group were 0.02 ., being under 0.05 , $\mathrm{H} 0$ was rejected and $\mathrm{H} 1$ accepted.

\section{Analytical methods}

Data was analyzed and figures plotted by means of an Excel package (Microsoft). Statistical analysis was performed with a SPSS package. Significant differences were taken at an alpha $=0.05$ for a T-Student test.

\section{Results}

Wnt3a increased its expression from the 5 cycles for 5 minutes, but especially from the 15 cycles and more even if the stimulus is performed for a longer time (40-90 minutes), as shown in figure 5. A high betacatenin expression is triggered during a 15 cycles and maintained for a longer period (40 to 90 minutes), as shown in figure 6, which might be pointing out a threshold effect.

Thus, there is a clear difference between 5 minutes and 40 minutes, without significance between 40 and 90 minutes. It also detects a threshold effect for Wnt-3a for the frequency of stimulation, although less defendant, between 1 and 5 cycles. Also and this is as important or more than the above, detected a minimum stimulus needed for activation of the pathway; below those thresholds defined above, there are no detectable differences with the control in the case of beta-catenin and minimal in the Wnt 3a duration of stimulation and in terms of the frequency in the case of Wnt-3a

\section{Discussion}

Research on the Wnt pathway has produced a high number of publications related to several fields ranging from tissue engineering to Cancer (A mechanical checkpoint controls multicellular growth through YAP/TAZ regulation by actin-processing factors [5]; Wnt signaling in cartilage development and diseases: lessons from animal studies [6]. Plenty of methods have been developed to study cell mechanotransduction, usually based in the culture of mesenchymal cells in membranes which are cyclically stretched (in one or two perpendicular axes), to induce a deformation to these cells, which could be interpreted as an epigenetic response leading to WNT activation. Even the vibration of the membranes of a loudspeaker has been shown to be an effective stimulus

Most often working with frequencies of stimulation around the $\mathrm{Hz}$ or less $[5,6]$, in this sense, this paper has worked with lower frequencies, an order of magnitude of cycles/minute, no $\mathrm{Hz}$ (cycle per second); What is also a certain novelty and may have increased sensitivity to detect this effect threshold and the minimum stimulus needed, not referred to in our knowledge in the literature. Sometimes the difference is not so great, since $0.3 \mathrm{~Hz}$ [6] and 15 stimuli per minute are a similar regime. It seems that the deformation of the cell is the most important stimulus, encrypting this around $10 \%[5,7]$ as ideal; with some data that say that even this level can be high [8], being $15 \%$ already clearly too [6]. Another aspect studied is the stimulation uni or bi-directional cell orientation is thus one of the most defining signals of cell stimulation by mechanical forces arising from their interaction, mechanical, with its surroundings: this is known from the work of Curtis [1] and was of the first findings that suggested the influence of the mechanical forces on the cell stimulation.

This directionality appears to be influenced by the rigidity of the substrate on which is cultivated cells, decreasing the ability of mechanical activation if the rigidity of the substrate (membrane that is cultivated) decreases [7]. Alterations at the nuclear level in perpendicular stimulation to cells grown in LPs (default directionality) not happening if any protein of the nuclear matrix [9] blocks have also been described via Wnt/beta-catenin demonstrates a synergism of stimulation with chemical factors in the culture medium $[5,10,11]$, with some performance of LRP5 [11], although there are other publications

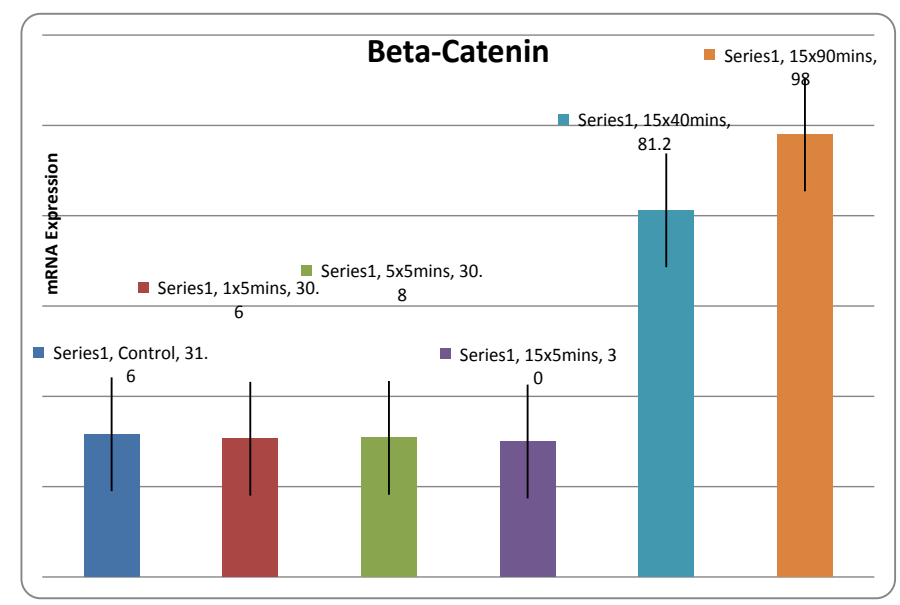

Figure 6. Beta-Catenin expression is found in higher proportions during long stimulation times. T-Student test $\mathrm{n}=8$ and $\alpha=0.05, \mathrm{H} 0: \mu 1=\mu 2 ; \mathrm{H1}: \mu 1>\mu 2$. P-values for the $5 \times 5$ and $15 \times 5$ group were 0.03 , being under $0.05, \mathrm{H} 0$ was rejected and $\mathrm{H} 1$ accepted. 
remaining prominence to LRP5 [10] , it has even been said that they are independent of Dkk-1, mechanical stimulation of beta-catenin routes, what has interest as Dkk-1 inhibitors are a way that had been taken and looks very promising, to generate drugs for treatment of osteoporosis.

In our results we seem to provoke a triggering response to flow in gene expression, however, it should be studied with more profusion in order to provide clear evidence which could be correlated with other experiments. Certainly a cell will not respond the same to different stimuli or forces. It must be noted that also that the research could provide an interesting insight for future studies where culture substrates with different elastic moduli could be implemented as a new variable of the study $[12,13]$.

No fits infer an accumulation effect simply for as long, since between 40 and 90 minutes just there is an increase, this being clearly manifest between the stimulation for 5 and for 40 minutes.

There are several differences between our work and the majority of publications, which could explain the novelty of our findings: the frequency of stimulation (already commented), but also the duration (maximum 90 minutes against days in many works) and the type of force used (hydrostatic compression instead of substrate stretch). This work made a stimulus of compression on hard scaffold, not a stretch of a membrane, believing that both bone and intervertebral disk environment is one cyclic compression.

Another important difference is that the gel was compressed, instead of stretching a membrane; while the transduction of the compression of gel on the cell really is in all likelihood a distraction of this deformation. Besides, to our knowledge, a threshold for mechanical stimulation had never been shown. There are many questions to be clarified in terms of the mechanical stimulation of the cells, arising directly from the results this work, for instance, keying that threshold in terms of duration of the stimulus effect.

\section{References}

1. Curtis AS (1964) The mechanism of adhesion of cells to glass. a study by interference reflection microscopy. J Cell Biol 20: 199-215. [Crossref]

2. Ingber DE (2003) Mechanobiology and diseases of mechanotransduction. Ann Med 35 564-577. [Crossref]

3. Nusse R, van Ooyen A, Cox D, Fung YK, Varmus H (1984) Mode of proviral activation of a putative mammary oncogene (int-1) on mouse chromosome 15. Nature 307: 131136. [Crossref]

4. Gnecchi M, Melo LG (2009) Bone marrow-derived mesenchymal stem cells: isolation, expansion, characterization, viral transduction, and production of conditioned medium. Methods Mol Biol 482: 281-294. [Crossref]

5. Aragona M, Panciera T, Manfrin A, Giulitti S, Michielin F, et al. (2013) A Mechanica Checkpoint Controls Multicellular Growth through YAP/TAZ Regulation by ActinProcessing Factors. Cell 154: 1047-1059. [Crossref]

6. Xiao W, Wang Y, Pacios S, Li S, Graves DT (2015) Cellular and Molecular Aspects of Bone Remodeling. Front Oral Biol 18: 9-16. [Crossref]

7. Thorpe SD, Buckley CT, Vinardell T, O’Brien FJ, Campbell VA, et al. (2010) The response of bone marrow-derived mesenchymal stem cells to dynamic compression following TGF-beta3 induced chondrogenic differentiation. Ann Biomed Eng 38: 28962909. [Crossref]

8. Steinmetz NJ, Bryant SJ (2011) The effects of intermittent dynamic loading on chondrogenic and osteogenic differentiation of human marrow stromal cells encapsulated in RGD-modified poly(ethylene glycol) hydrogels. Acta Biomater 7: 3829-3840. [Crossref]

9. Throm Quinlan AM, Sierad LN, Capulli AK, Firstenberg LE, Billiar KL (2011) Combining dynamic stretch and tunable stiffness to probe cell mechanobiology in vitro. PLoS One 6: e23272. [Crossref]

10. Kearney EM, Prendergast PJ, Campbell VA (2008) Mechanisms of strain-mediated mesenchymal stem cell apoptosis. J Biomech Eng 130: 061004. [Crossref]

11. Li Y, Chu JS, Kurpinski K, Li X, Bautista DM, et al. (2011) Biophysical regulation of histone acetylation in mesenchymal stem cells. Biophys $J$ 100: 1902-1909. [Crossref]

12. Case N, Ma M, Sen B, Xie Z, Gross TS, et al. (2008) Beta-catenin levels influence rapid mechanical responses in osteoblasts. J Biol Chem 283: 29196-29205. [Crossref]

13. Robinson JA, Chatterjee-Kishore M, Yaworsky PJ, Cullen DM, Zhao W, et al. (2006) $\mathrm{Wnt} /$ beta-catenin signaling is a normal physiological response to mechanical loading in bone. J Biol Chem 281: 31720-31728. [Crossref]

Copyright: ${ }^{2} 2016$ Díez-Ulloa MA. This is an open-access article distributed under the terms of the Creative Commons Attribution License, which permits unrestricted use, distribution, and reproduction in any medium, provided the original author and source are credited. 